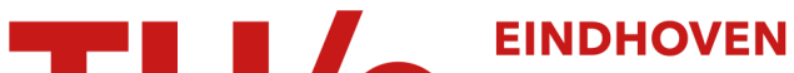 \\ UNIVERSITY OF \\ TECHNOLOGY
}

\section{Self-organisation of semi-flexible rod-like particles}

\section{Citation for published version (APA):}

De Braaf, B., Oshima Menegon, M., Paquay, S., \& van der Schoot, P. P. A. M. (2017). Self-organisation of semiflexible rod-like particles. Journal of Chemical Physics, 147(24), [244901]. https://doi.org/10.1063/1.5000228

DOI:

$10.1063 / 1.5000228$

Document status and date:

Published: 28/12/2017

\section{Document Version:}

Publisher's PDF, also known as Version of Record (includes final page, issue and volume numbers)

\section{Please check the document version of this publication:}

- A submitted manuscript is the version of the article upon submission and before peer-review. There can be important differences between the submitted version and the official published version of record. People interested in the research are advised to contact the author for the final version of the publication, or visit the $\mathrm{DOI}$ to the publisher's website.

- The final author version and the galley proof are versions of the publication after peer review.

- The final published version features the final layout of the paper including the volume, issue and page numbers.

Link to publication

\section{General rights}

Copyright and moral rights for the publications made accessible in the public portal are retained by the authors and/or other copyright owners and it is a condition of accessing publications that users recognise and abide by the legal requirements associated with these rights.

- Users may download and print one copy of any publication from the public portal for the purpose of private study or research.

- You may not further distribute the material or use it for any profit-making activity or commercial gain

- You may freely distribute the URL identifying the publication in the public portal.

If the publication is distributed under the terms of Article 25fa of the Dutch Copyright Act, indicated by the "Taverne" license above, please follow below link for the End User Agreement:

www.tue.nl/taverne

Take down policy

If you believe that this document breaches copyright please contact us at:

openaccess@tue.nl

providing details and we will investigate your claim. 


\section{Self-organisation of semi-flexible rod-like particles}

Bart de Braaf, Mariana Oshima Menegon, Stefan Paquay, and Paul van der Schoot

Citation: The Journal of Chemical Physics 147, 244901 (2017);

View online: https://doi.org/10.1063/1.5000228

View Table of Contents: http://aip.scitation.org/toc/jcp/147/24

Published by the American Institute of Physics

\section{Articles you may be interested in}

Perspective: Dissipative particle dynamics

The Journal of Chemical Physics 146, 150901 (2017); 10.1063/1.4979514

Ordering nanoparticles with polymer brushes

The Journal of Chemical Physics 147, 224901 (2017); 10.1063/1.5006048

Motion of a Janus particle very near a wall

The Journal of Chemical Physics 147, 224906 (2017); 10.1063/1.4994843

Crystallization of Lennard-Jones liquids under dynamic compression: Heterogeneous and homogeneous nucleation

The Journal of Chemical Physics 147, 244501 (2017); 10.1063/1.5010088

Construction of non-Markovian coarse-grained models employing the Mori-Zwanzig formalism and iterative Boltzmann inversion

The Journal of Chemical Physics 147, 244110 (2017); 10.1063/1.5009041

The Landau-de Gennes approach revisited: A minimal self-consistent microscopic theory for spatially inhomogeneous nematic liquid crystals

The Journal of Chemical Physics 147, 244505 (2017); 10.1063/1.4993574

\section{AIP $\left.\right|^{\text {The Journal of }}$ AIP $\mid$ chemical Physics} PERSPECTIVES 


\title{
Self-organisation of semi-flexible rod-like particles
}

\author{
Bart de Braaf, ${ }^{1}$ Mariana Oshima Menegon, ${ }^{1, a)}$ Stefan Paquay, ${ }^{1, b)}$ and Paul van der Schoot ${ }^{1,2}$ \\ ${ }^{1}$ Department of Applied Physics, Eindhoven University of Technology, P.O. Box 513, 5600 MB Eindhoven, \\ The Netherlands \\ ${ }^{2}$ Institute for Theoretical Physics, Utrecht University, Princetonplein 5, 3584 CC Utrecht, The Netherlands
}

(Received 14 August 2017; accepted 11 December 2017; published online 28 December 2017)

\begin{abstract}
We report on a comprehensive computer simulation study of the liquid-crystal phase behaviour of purely repulsive, semi-flexible rod-like particles. For the four aspect ratios we consider, the particles form five distinct phases depending on their packing fraction and bending flexibility: the isotropic, nematic, smectic A, smectic B, and crystal phase. Upon increasing the particle bending flexibility, the various phase transitions shift to larger packing fractions. Increasing the aspect ratio achieves the opposite effect. We find two different ways in which the layer thickness of the particles in the smectic A phase may respond to an increase in concentration. The layer thickness may either decrease or increase depending on the aspect ratio and flexibility. For the smectic B and the crystalline phases, increasing the concentration always decreases the layer thickness. Finally, we find that the layer spacing jumps to a larger value on transitioning from the smectic A phase to the smectic B phase. Published by AIP Publishing. https://doi.org/10.1063/1.5000228
\end{abstract}

\section{INTRODUCTION}

Rod-like colloidal particles, DNA strands, carbon nanotubes, and filamentous viruses have in common that, if dispersed in a fluid at sufficiently high concentrations, they exhibit various kinds of liquid-crystalline phases. This is because with increasing concentration, the dispersion runs out of free volume leading to increasingly ordered states. This class of material is usually referred to as lyotropic liquid crystals, which sets them apart from thermotropic liquid crystals because the driving force is not energy but, in essence, entropy. This was first recognised by Lars Onsager in his seminal paper describing the isotropic-to-nematic phase transition of cylindrical particles interacting via a hard-core repulsive interaction. ${ }^{1}$ In agreement with experiment, the theory predicts the transition to occur at a volume fraction that decreases inversely proportional to the aspect ratio of the particles. The impact of the particle bending flexibility on the isotropic-nematic transition was first investigated theoretically by Khokhlov and Semenov more than thirty years later, ${ }^{2,3}$ a decade after others investigated how flexibility impacts upon the nematic-columnar and the nematic-smectic A transitions. ${ }^{4-7}$

Over the past few decades, interest in lyotropic liquid crystals has increased significantly, in part because of potential applications and in part because of the development of wellcontrolled model particles. ${ }^{8,9}$ Indeed, lyotropic liquid crystals have been intensively investigated experimentally, ${ }^{10-14}$ theoretically ${ }^{15-19}$ and with the aid of computer simulations. ${ }^{20-26}$ In spite of this, our understanding of the isotropic and nematic phases is most comprehensive, whilst that of the others remains

\footnotetext{
a)Author to whom correspondence should be addressed: m.oshima. menegon@tue.nl

b)Present address: Department of Physics, Brandeis University, 415 South St., Waltham, Massachusetts 02453, USA.
}

much less detailed. In particular, how the flexibility and aspect ratio impact upon the other liquid crystal transitions has received much less attention. Here, we aim to fill in this gap from the perspective of computer simulations, in particular, because these are much more difficult to address theoretically. One reason is that the second virial approximation, which allowed Onsager to accurately describe the isotropic-nematic transition, no longer holds at densities where the smectic and columnar phases appear. Another reason is translationrotation coupling, which makes density functional and integral equation theories virtually intractable. $7,18,27$

We extend earlier simulation studies on semi-flexible chains by covering a larger range in persistence length, aspect ratio, and particle numbers and investigate more comprehensively the microscopic structure of the liquid crystalline and crystalline phases. In agreement with theory and simulation, we find that particles with longer aspect ratio support over a larger concentration range and a broader range of bending flexibilities liquid-crystalline states. This is particularly true for the nematic and the smectic A phases. We find that the stability of the smectic B and crystalline phases, recently both found experimentally in colloidal systems, ${ }^{12,13}$ to be less sensitive to both the aspect ratio and flexibility, at least for the ranges investigated. The aspect ratio of our particles varied between 6 and 11 while the ratio of the bare contour length and persistence length varied between 0.05 and 0.5 . For these aspect ratios and persistence lengths, all phase transitions are either second order or weakly first order, except the transition between smectic A and smectic B that clearly is first order. The difference in behaviour of the smectic A and B phases expresses itself most clearly in how the layer spacing responds to increases in density. For the smectic B phase, the layer spacing always decreases with increasing density. This is not so for the smectic A phase, where depending on the aspect ratio and persistence length, it may increase or decrease, 
depending on whether the increase of the particles density is translated into reduced layer spacing and/or increased in-layer density.

The remainder of this paper is structured as follows. In Sec. II, we describe our model particles that we construct from overlapping, mutually repulsive bead-spring chains. We also make explicit our simulation protocol and explain how we identify the various liquid crystal phases in our simulation data. In Sec. III A, we present the phase diagrams we obtain and show how the aspect ratio and flexibility influence the phase transitions. In Sec. III B, we describe the influence of the phase transitions on the individual particle structure and smectic layer thickness. We furthermore present a simple model based on the Onsager theory that explains the changes in the particle length as a function of density in the isotropic and nematic phases in the Appendix. Finally, in Sec. IV, we present our most important conclusions.

\section{METHODS AND ANALYSIS}

To study the equilibrium properties of semi-flexible rodlike particles, we perform MD simulations on 4608 beadspring chains consisting of $n$ beads of mass $m$ using the software package LAMMPS. ${ }^{28}$ Within a chain, consecutive pairs of beads interact via a harmonic potential $U_{r}=1 / 2 \kappa\left(r-r_{0}\right)^{2}$, where $\kappa$ is the force constant and $r_{0}$ is the rest distance between their centres of mass corresponding to half of the bead diameter $D$. See Fig. 1 . Hence, the beads partially overlap in order to provide a smoother particle surface and to prevent biased stacking between the rod-like particles in highly congested phases. A harmonic bending potential $U_{\theta}=1 / 2 \kappa_{\theta}(\theta-\pi)^{2}$, where $\kappa_{\theta}$ is the bending constant, $\theta$ in radians, is assigned to consecutive bonds between beads to model bending stiffness. Except for the nearest neighbour beads along the same chain, all beads interact via a purely repulsive, truncated, and shifted Lennard-Jones (LJ) potential $U=4 \epsilon\left[(r / D)^{-12}-(r / D)^{-6}\right]+\epsilon$ for $r \leqslant 2^{1 / 6} D$ and $U=0$ for $r>2^{1 / 6} D$, with $\epsilon=k_{B} T$, where $k_{B}$ is the Boltzmann constant and $T$ is the absolute temperature. The effective hard sphere diameter of a bead along the chain $D_{\text {eff }}$ is slightly larger than $D: D_{\text {eff }} \sim 1.017 D$. This estimate can be obtained by comparing the second virial coefficient for our purely repulsive soft potential to the second virial coefficient for the hard sphere potential.

We perform isobaric-isothermal (NPT) simulations at various pressures. To control the temperature and pressure in our

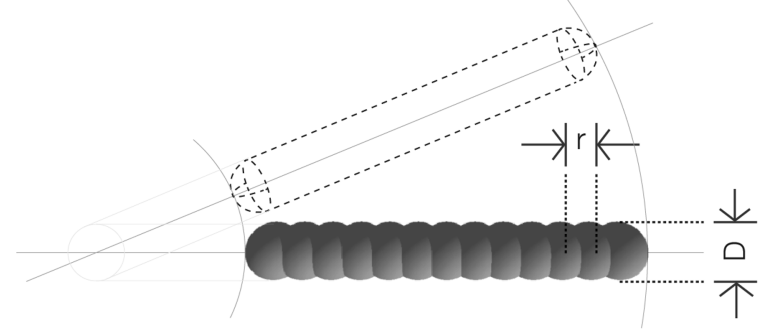

FIG. 1. Schematic representation of the semi-flexible rod-like particles. They are modeled as a bead-spring chain with diameter $D$. The beads partially overlap for a smoother surface, allowing closer comparison with spherocylinders (dashed schematic). The rest distance $r_{0}$ of the harmonic bond potential corresponds to half the bead diameter. simulations, we employ the Nosé-Hoover thermostat and barostat. The thermal energy $k_{B} T$ is our reference energy unit. The barostat can adjust the rectangular simulation box dimensions independently, which allows relaxation to the correct layers spacings in the smectic and crystalline phases. Therefore the changes in the box shape are anisotropic. Each simulation runs for 20000 time units. Our time unit is set by the Lennard Jones time scale $\left(m / k_{B} T\right)^{1 / 2} \sigma$, and we use time steps of $10^{-3}$ in these units in our simulations, implying that our simulations run $2 \times 10^{7}$ time steps. The relaxation times for temperature and pressure are 0.01 and 0.1 time units corresponding to about 10 and 100 time steps, respectively. Approximately 200 configurations of every run are stored, i.e., one every 100 time units.

The initial configuration is that of the crystal phase, with all rod-like particles perfectly aligned, AAA stacked in 16 layers with $18 \times 16$ particles each, i.e., the layers are identical copies shifted along the director, and in each layer, there is perfect hexagonal ordering. That the initial box is very elongated is sensible because the particles themselves have a large aspect ratio. In the isotropic phase, the box elongation relaxes and on average becomes isometric albeit the box shape fluctuates considerably, in particular, near the isotropic-to-nematic phase transition. In the nematic phase, the box can become very much more elongated than the initial elongation. In the smectic and crystalline phases, the box anisometry remains roughly equal to the initial one. If in our simulations, one box dimension drops below about one particle length, we discard the run.

The reason why we take the crystal phase as our initial configuration is that starting from an ordered structure, we are unlikely to end up in a jammed state. Arguably, the lower the symmetry of the configuration of particles is, the more likely it jams on account of the associated decrease in free volume. Of course, one could argue that this procedure might give rise to metastable layered phases, that is, the smectic A and $\mathrm{B}$ phases and the crystalline phase. To verify that this is not the case, we performed compression simulations for one particular aspect ratio and one degree of flexibility near all phase transitions that we find in our simulations starting off the crystalline state. For this purpose, we use a configuration from the highest concentration of the less ordered phase and increase the pressure to a value at which the more ordered phase should appear. We then evolved the system in all cases by $2 \times 10^{7}$ time steps and evaluated the order parameters (see below). The order in which the transitions appear is consistent with the expansion simulations, and the largest disagreement in the concentration at which the transitions occur is $0.5 \%$. Hence, we believe that the results of our simulations starting off the crystal configuration are robust.

We set the elastic constant $\kappa$ at a large value of $100 k_{B} T / \sigma^{2}$ to ensure minimal entropic stretching of the bonds. In other words, the average bond length is very close to the rest bond length of one-half $\sigma$. In our simulations, we allow for chains consisting of $n=13,15,17$, and 21 beads per chain. We find the corresponding aspect ratios $L_{0} / D$ in the limit of zero pressure to be $L_{0} / D=6.46,7.54,8.62$, and 10.77 . For every aspect ratio, we vary the bending stiffness $\kappa_{\theta}$ to obtain a series of ratios of the contour length $L_{0}$ and the persistence length $L_{p}$, 


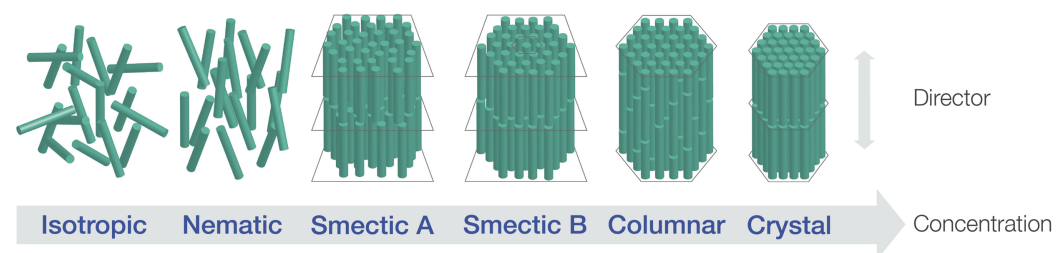

FIG. 2. Schematic representation of the liquid crystal phases found in aqueous dispersions of the rod-like fd virus. ${ }^{13}$ The phase sequence with increasing concentration is isotropic, nematic, smectic A, smectic B, columnar, and crystal. The double pointed arrow indicates the preferential direction of the aligned particles, the director. We note that the precise structure of the crystal phase is unknown.

$L_{0} / L_{p}=0.05,0.1,0.2,0.3,0.4$, and 0.5 . The persistence length we calculated from the equality $L_{p}=\kappa_{\theta} r / k_{B} T$ is valid for an infinitely large number of beads and $\kappa_{\theta} r^{2} / k_{B} T \gg 1 .^{25}$

To calculate the corresponding volume fraction $\phi$ of any given configuration, we take the equilibrated volume of the simulation box $V$ for a given pressure $P$ and define the volume occupied by the particles as the fixed number of chains $N$ in the system times the occupied volume $v_{0}$ by each chain, so $\phi=N v_{0} / V$. We approximate the volume of a chain by taking a spherocylinder with volume $v_{0}=\pi D^{3} / 6+\pi D^{2} L_{0} / 4$.

The equilibrium configurations stored are used to calculate the usual order parameters and the pair correlation functions of the collection of particles. The order parameters quantify (1) the degree of orientation of the chains, given by the nematic order parameter $S_{2}$; (2) the organisation in layers perpendicular to the director, given by the smectic order parameter $\tau$; and (3) the hexagonal ordering of the closest neighbours within the same layer, which is described by the bond order parameter $\psi_{6} \cdot{ }^{29-31}$ With these order parameters, the isotropic, nematic, smectic A, columnar, and smectic B or crystal phases can be identified and distinguished. All these phases are schematically represented in Fig. 2.

In the isotropic phase, there is only a short-range correlation between the positions and between the orientations of the chains, and $S_{2}$ should be zero, but need not be in account of finite size effects. With the alignment of the particles in the nematic phase, the order parameter $S_{2}$ increases abruptly when crossing the phase boundary, so it can be readily identified. The

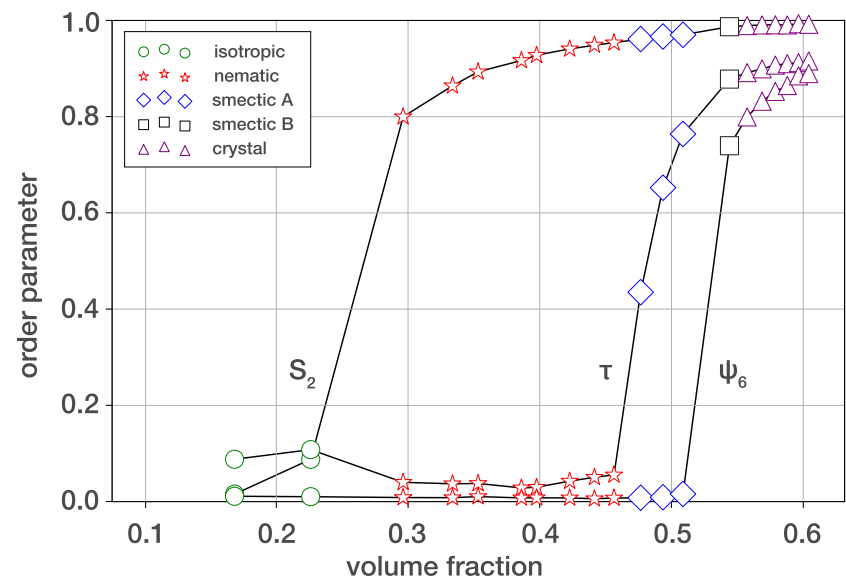

FIG. 3. Order parameters as a function of the volume fraction $\phi$ for aspect ratio $L_{0} / D=10.77$ and flexibility $L_{0} / L_{p}=0.05$. The nematic order parameter is $S_{2}$, the smectic order parameter is $\tau$, and the bond order parameter is $\psi_{6}$. same is true for the smectic and bond order parameters $\tau$ and $\psi_{6}$, allowing us to identify the smectic A phase and the smectic B or crystal phase. See Fig. 3. Snapshots of the various phases are given in Fig. 4. The smectic B phase differs from the smectic $\mathrm{A}$ and crystal phases in that it does have (quasi) long-range in-plane bond order but (quasi) no actual long-range positional order. The smectic A phase has neither, and the crystal phase has both long-range bond order and positional order. We cannot distinguish between the smectic B and crystal phase based only on calculating the correlation function $g_{6}^{\text {lay }}$ of the order parameter $\psi_{6}$ due to the finite size of the system. See Fig. 4 . However, we can distinguish between them by considering the in-layer pair correlation function $g^{\text {lay }}$ of the centres of mass of the chains. ${ }^{25,32}$

As can be seen in Figs. 5(a) and 5(b), there is a clear difference in $g^{\text {lay }}$ between two states at different pressures with equal magnitude of the order parameters $S_{2}, \tau$, and $\psi_{6}$. Figure 5(a) exhibits a split second peak in $g^{\text {lay }}$, a characteristic of a crystalline phase that the system with the pressure shown in Fig. 5(b) does not have. We therefore associate the absence of peak splitting with the smectic B phase and assume that the phase transition takes place when the second peak in the in-layer pair correlation function splits. So, we use the splitting of the second peak in $g^{\text {lay }}$ as a proxy for distinguishing between the smectic $\mathrm{B}$ and the crystal phases. Note that the smectic B phase that we identify in Fig. 5(b) has a much noisier $g_{6}^{\text {lay }}$ than that of the crystal phase of Fig. 5(a).
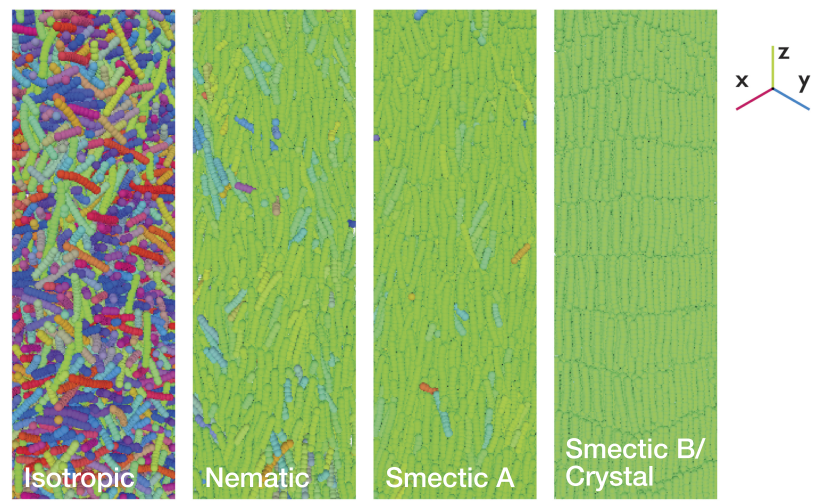

FIG. 4. Snapshots representing the arrangement of particles along the director for the different phases observed in our simulations for aspect ratio $L_{0} / D$ $=6.46$ and ratio of contour length and persistence length of $L / L_{p}=0.1$. From left to right with increasing density: isotropic, nematic, smectic $\mathrm{A}$, and smectic $\mathrm{B} /$ crystal. The colour of the particle is a combination of red, blue, and green, whose intensities are, respectively, proportional to the $x, y$, and $z$ components of the orientation of the particle. 
(a)
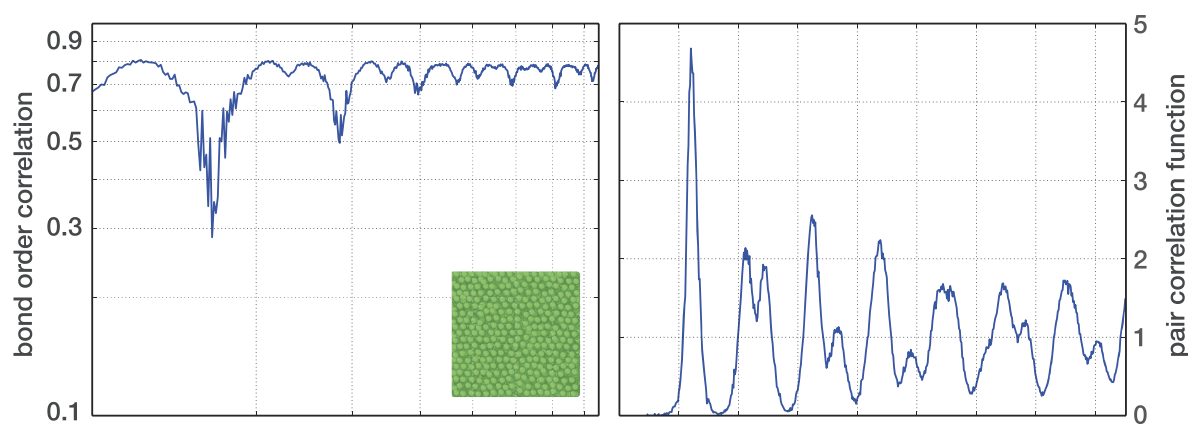

(b)

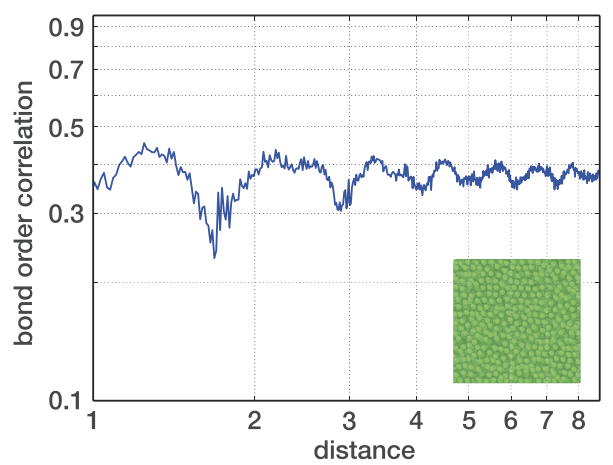

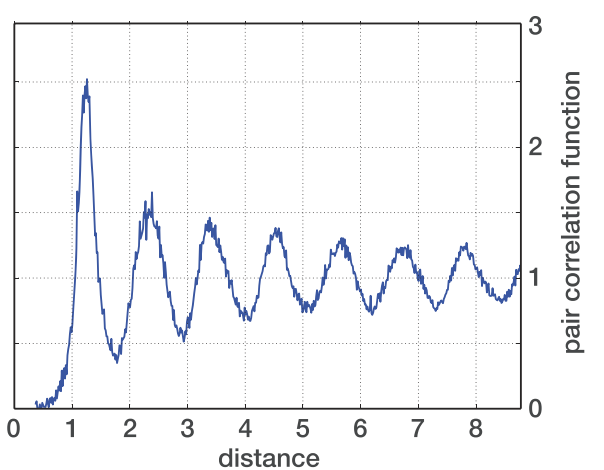

FIG. 5. (a) The $\psi_{6}$ correlation function $g_{6}^{\text {lay }}$ (left) and the in-layer pair correlation function $g^{\text {lay }}$ (right) as a function of the distance the centers of mass of the rods (in units of rod thickness). The particles have an aspect ratio $L_{0} / D=10.77$, flexibility $L_{0} / L_{p}$ $=0.5$, and volume fraction $\phi=0.63$. The pair correlation function exhibits peaks characteristic for a crystal phase, and hence we identify it as such. (b) The same for a volume fraction of $\phi$ $=0.57$. The pair correlation function does not show the characteristic crystal peaks. Hence at a volume fraction of $\phi=0.57$, the particles must in the smectic B phase. See also the main text.
The difference in the structure of the crystal and smectic B phases is also evident from the snapshots also presented in Fig. 5.

In order to determine the crystal symmetry, we compare the pair correlation function of the centers of mass of the particles. We distinguish four cases. In the first case, we calculate the pair correlation function for particles in the same layer. In the second case, we consider pairs of particles in consecutive layers. In the third and fourth cases, the pair correlation function considers pairs of particles separated by one and two layers. We expect that all pair correlation functions must be similar for the AAA crystal structure, whilst the first and fourth cases should be similar for the ABC structure. We observe neither of these patterns, implying that we cannot pinpoint the exact crystal structure. A possible explanation for this is that the ordering between layers is not so well defined for semi-flexible particles. The fact that we start off from an initial AAA structure that does not seem to survive concludes that our simulations are not kinetically trapped.

\section{RESULTS AND DISCUSSION}

\section{A. Phase diagrams}

The phase diagrams of our particles are presented in Fig. 6. Recall that our particles are semi-flexible, rod-like chains interacting via a soft-core, repulsive potential. We present phase diagrams as a function of volume fraction and bending flexibility, ranging from $L_{0} / L_{p}=0.05$ to 0.5 , covering particles from near the rigid-rod limit to semi-flexible chains, for four aspect ratios, $L_{0} / D=6.46,7.54,8.62$, and 10.77 . We distinguish between the following phases: isotropic, nematic, smectic A and smectic B/crystal (Fig. 4). For our set of parameters, we did not encounter any evidence for a columnar phase. Based on what we know on the phase behaviour of the fd virus, which does support a columnar phase, we must conclude that our particles do not have a large enough aspect ratio for this phase to appear in the phase diagram. ${ }^{14}$

Focusing on the aspect ratio $L_{0} / D=6.46$ first and representing the trends observed for the other aspect ratios, Fig. 6 tells us that all phase transitions shift to larger volume fractions with increasing flexibility. The isotropic-nematic transition increases approximately linearly with increasing degree of flexibility, which for large persistence lengths is consistent with theory and Monte Carlo. ${ }^{20}$ Both the isotropic-nematic and the nematic-smectic A transitions are significantly impacted upon by any bending flexibility. Theoretically, this has been predicted to be the case albeit these theories are typically valid in the long-chain and/or large persistence length limits relative to the width of the particles. ${ }^{2,3,6,7,15-17}$ The result also agrees with previous simulation by Bladon and Frenkel. ${ }^{22}$ We find that the smectic A phase is strongly destabilised by decreasing the chain stiffness, in line with results from earlier computer simulations by Cinacchi and Gaetani on shorter rods and for smaller box sizes. ${ }^{23}$

For values of $L_{0} / L_{p}>0.1$, we find a direct transition from the nematic phase to the smectic $B$ phase, i.e., the smectic A phase disappears for large enough flexibilities. We notice that the transitions between the nematic and the smectic $\mathrm{B}$, the smectic A and the smectic B, and the smectic B and the crystal phases are much less sensitive to changes in the particle flexibility, and, in fact, to variations in the aspect ratio. The smectic A phase is more stable for larger aspect ratios and present in the phase diagram for all flexibilities probed for the aspect ratio $L_{0} / D=10.77$. The transition from the smectic A or nematic to the smectic B and that from the smectic B to the crystal phase is only very weakly dependent on the aspect ratio and bending flexibility of the particles. This is not entirely unexpected, on the one hand, because the particles in these dense phases are almost perfectly aligned and, on the other hand, because the Odijk deflection length $\lambda_{\text {Odijk }}=L_{p}\left\langle\theta^{2}\right\rangle$ turns out to be of the 

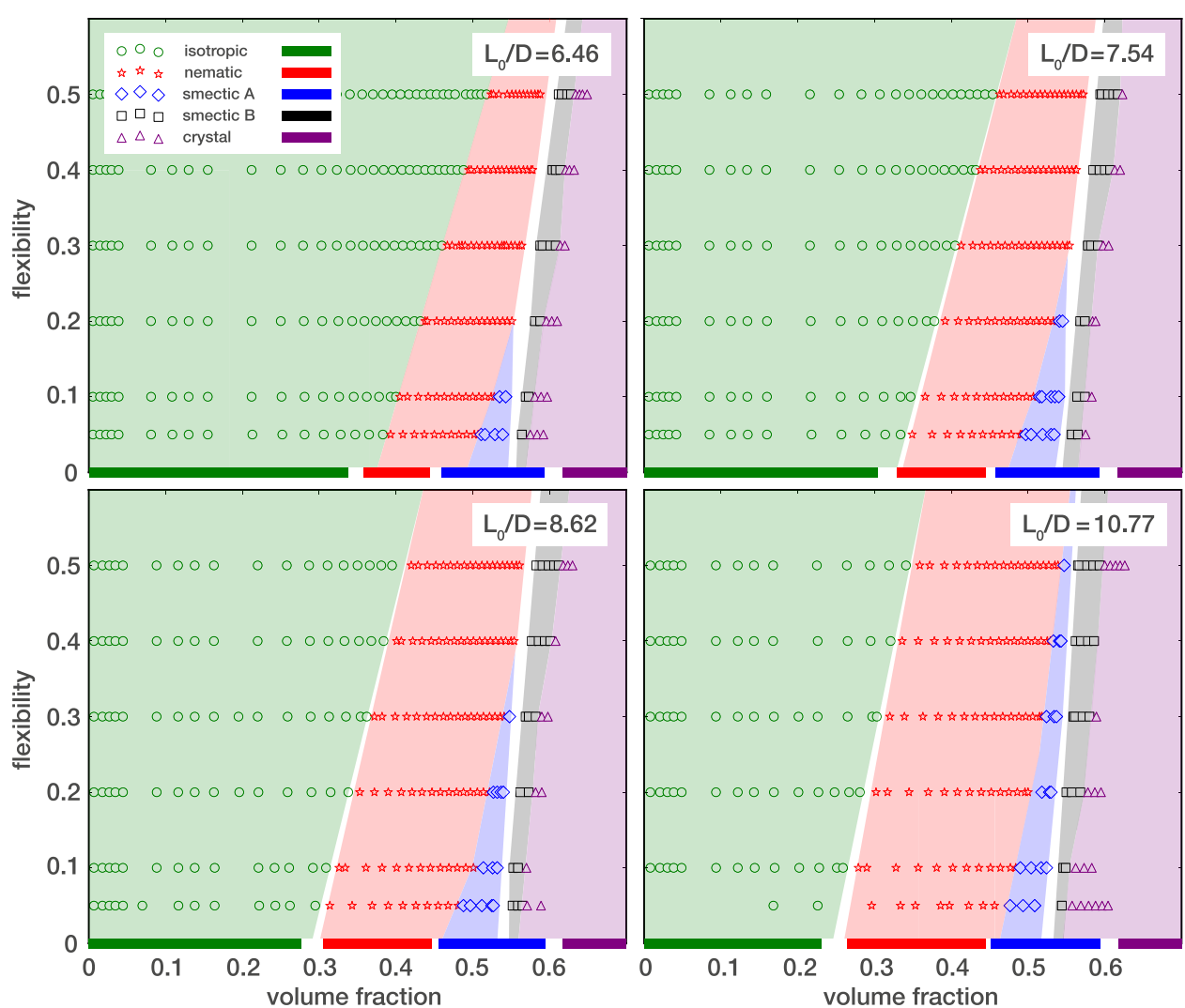

FIG. 6. Phase diagrams as a function of the volume fraction $\phi$ and flexibility $L_{0} / L_{p}$ for rods with aspect ratios $L_{0} / D=6.46,7.54,8.62$, and 10.77. Indicated are the isotropic phase (green circles), the nematic phase (red stars), the smectic A phase (blue diamonds), the smectic B phase (black squares), and the crystal phase (purple triangles). Corresponding background colours are added to aid identifying the various phases. The isotropic-nematic and the nematicsmectic A phase transitions shift to higher volume fractions with increasing degree of flexibility. Furthermore the smectic A phase disappears above a critical, aspect-ratio-dependent degree of flexibility. The smectic A-smectic B or nematic-smectic B and smectic Bcrystal phase transitions shift to larger volume fractions with increasing degree of flexibility albeit the effect is relatively weak. The bars placed at zero flexibility indicate the simulation results of Bolhuis and Frenkel for infinitely rigid, hard spherocylinders for comparison. ${ }^{33}$ order of the width of the particles in those phases. This implies that bending modes with smaller wavelengths cannot be suppressed, and that in this limit, the bending flexibility should be unimportant. ${ }^{15}$ Practically, this is true if the degree of alignment of particles, given by the nematic order parameter $S$, is larger than $1-(3 D) /\left(2 L_{p}\right)$. This happens to be the case for the smectic B and crystal phases for the range of flexibilities that we cover.

Our simulation results are consistent with those of Bolhuis and Frenkel for rigid, hard spherocylinders ${ }^{33}$ represented in Fig. 6 by the bars placed at zero flexibility $\left(L_{0} / L_{p} \rightarrow 0\right)$. The agreement is even quantitative for less ordered phases whilst for the highly ordered phases, the phase transitions in Bolhuis and Frenkel's simulations shift to larger concentrations compared to ours. There are several explanations for this. First, our rod-like chains are slightly compressible. As we shall see in Sec. III B, excluded-volume interactions cause the chains to compress in particular in the phases where free volume become scarce, so in the denser phases. Second, our particles interact through a soft-core interaction while the rigid rods of Ref. 33 interact via a hard-core potential. Third, our simulation box is much larger than that of the 1997 study of Bolhuis and Frenkel. Their particle number was at most 600 whilst in our case, it is 4608 , suggesting that finite size effects might also play a role in the discrepancy.

Regarding the order of the transitions, we can only confirm that the transition from the nematic or the smectic A to the smectic B phase is most definitely of first order: we observe a clear jump in the density at the pressure where the transition takes place (results not shown). We find the isotropic-to-nematic transition to be weakly first order, if at all, but it seems to become more strongly first order with increasing aspect ratio, to shift to lower concentrations and generally to become more stable. This is in line with the computer simulations of Bolhuis and Frenkel. ${ }^{33}$ For the other transitions, we find that, if there are jumps, we do not have the resolution to observe them. The experiments of, e.g., Grelet et al. on aqueous dispersions of fd virus particles, which have an aspect ratio 130, indicate that the nematic-to-smectic A transition is first order. ${ }^{11-13}$ The order of the transition from smectic A to smectic B for fd virus remains unclear. Fd virus does not transition from smectic B to crystal but to a columnar phase. ${ }^{12,13}$ We hypothesise that the large aspect ratio of the viruses particles somehow stabilises the columnar phase.

Having discussed the macroscopic (thermodynamic) properties of our particles, we next investigate in more depth how the particles and the structure of the more ordered phases respond to the particle length and flexibility. Interestingly, we find that the layer thickness in the smectic A phase may increase or decrease with increasing concentration depending on the flexibility and aspect ratio of the chain. This increase of the layer thickness with increasing density is counter-intuitive but, as we shall see next, it is somehow connected with the aspect ratio and flexibility.

\section{B. Microscopic structure}

Our first probe of the microscopic structure of the various phases is the actual contour length of the chains relative to the unperturbed contour length. This is important because our particles are not only flexible but also slightly compressible. Hence, we expect that with increasing particle density, they should become shorter in order to accommodate a decreasing 
free volume. This can be seen as a drawback of our model particles but in fact allows us to address the question to what extent the particle flexibility impacts upon the excluded volume in the isotropic phase, and vice versa if and how excluded volume interactions impact upon the effective particle bending flexibility.

In Fig. 7(a), the contour length $\langle L\rangle$ is scaled to the reference contour length $L_{0}$ for the aspect ratio $L_{0} / D=8.62$ as a function of the volume fraction and the flexibility. The contour length decreases with increasing volume fraction in the isotropic phase. This decrease does not depend on the particle flexibility suggesting that volume exclusion in the isotropic phase is an invariant of the particle flexibility, as has been presumed in the past. ${ }^{2,3,8,9,17}$ We observe a small but sudden increase of the contour length at the isotropic-nematic transition, except for the most flexible chains for which the transition seems to become either second order or very weakly first order. (We note that both the insensitivity of the excluded volume to chain flexibility in the isotropic phase and the lengthening of the rods in crossing over to the nematic phase was observed by Wilson, using a very different flexibility model in his simulations. ${ }^{34}$ )
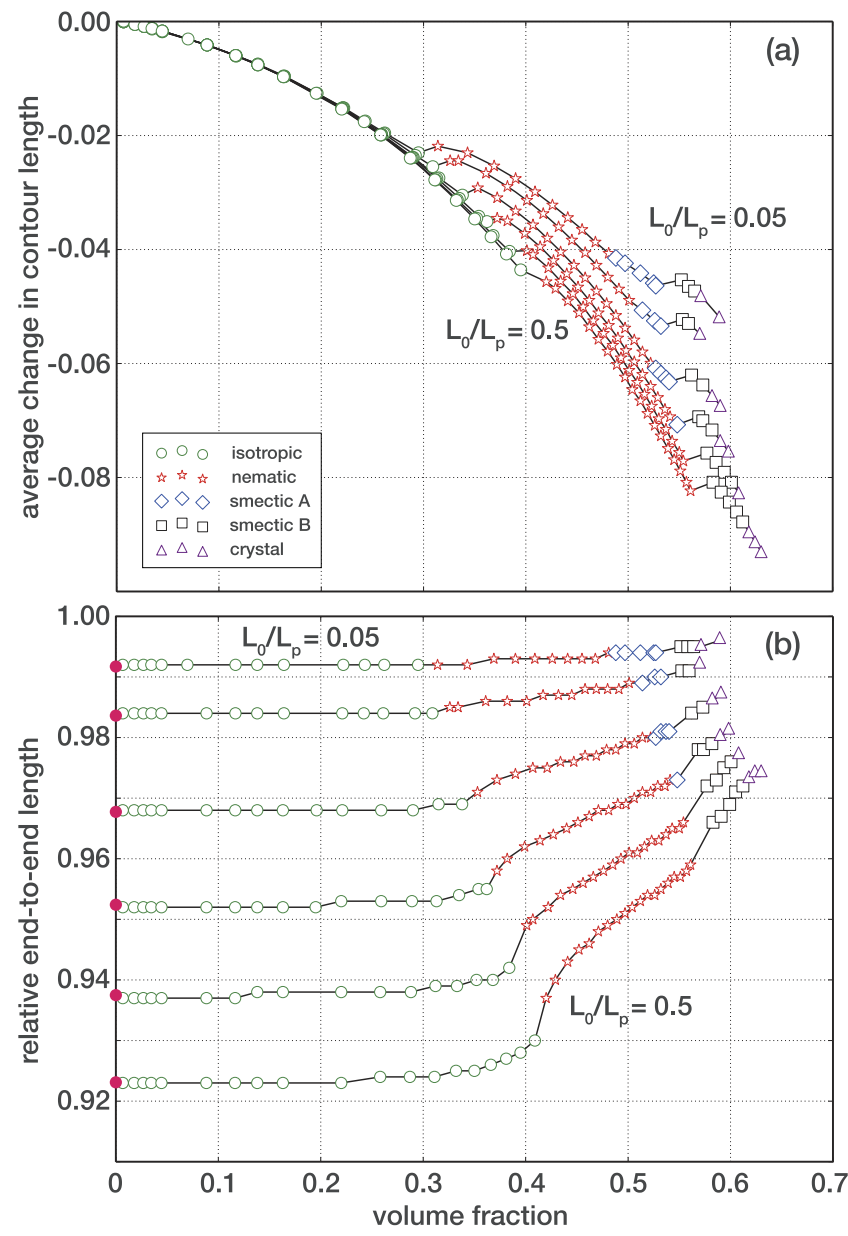

FIG. 7. (a) The average change in the contour length of the chains $\left(\langle L\rangle-L_{0}\right) / L_{0}$ as a function of the volume fraction $\phi$ for various aspect ratio $L_{0} / D=8.62$. The symbols are defined in Fig. 6. The compression and jumps in length are explained in the main text. (b) The relative end-to-end length $\left\langle L_{\text {ete }}\right\rangle\left\langle\langle L\rangle\right.$ as a function of the volume fraction $\phi$ for aspect ratio $L_{0} / D=8.62$. Filled circles in magenta represent the prediction for the worm-like chain model.
Arguably, the reason for this jump is an increased free volume caused by the alignment of the particles in the nematic phase. ${ }^{9}$ This confirms that the transition is first order albeit more weakly so for the more flexible chains. In the nematic phase, the contour length decreases with increasing concentration again because of the decrease in free volume with increasing concentration. We find a much stronger jump on going from the nematic or smectic A to the smectic B phase. Simple second virial calculations presented in the Appendix confirm the observed trends for the isotropic and nematic phases, explaining also the jump in length.

In Fig. 7(b), the end-to-end length $\left\langle L_{\text {ete }}\right\rangle$ is scaled to the measured contour length $\langle L\rangle$ for aspect ratio $L_{0} / D=8.62$ as a function of the volume fraction and the flexibility. In the isotropic phase, this end-to-end length apparently depends only on the ratio $L_{0} / L_{p}$. It depends weakly on the concentration except for the most flexible chains and then only near the isotropic-to-nematic phase transition. This we argue is again caused by the excluded-volume interactions not being affected by particle flexibility. Our measured values for the relative end-to-end length are in very good agreement with the prediction given by the worm-like chain model, also indicated in the figure. This confirms that our estimate of the persistence length for our model chains is accurate. In the liquid crystalline phases, the end-to-end distance does depend on the concentration and more so the more flexible the particles. This can straightforwardly be understood by realising that a combination of persistence and the molecular ordering field attenuates the bending fluctuations. ${ }^{15,34}$ The molecular ordering becomes stronger the larger the particle density. The same is true for the remaining phase transitions as in fact we already alluded to in Sec. III A.

Perhaps the most interesting structural feature is how the average smectic layer thickness depends on the contour length and persistence length of the particles that we calculate from the recipe of Ref. 30. In practice, the layer thickness corresponds to the layer height (rod length) plus the spacing between layers. This is shown in Fig. 8. For all cases, we find that while transitioning from the smectic A to the smectic B phase, the layer thickness increases. We speculate that this is due to the larger degree of in-layer packing possible in the more strongly ordered smectic B phase. In essence, this is caused by an increase in free volume. Depending on the aspect ratio and flexibility, we observe that the layer thickness in the smectic A phase itself may increase or decrease with increasing concentration. This is not so for the smectic B and crystalline phases. It seems that in the smectic A phase, increasing the particle density may translate into a more or less proportional increase in the in-layer density. If the in-layer density increases more strongly than the average density, then the layer thickness must increase. Because of the appreciable scatter in the data, we have not been able to find a clear trend. We also have no explanation for this phenomenon.

We notice that measurements of the concentration dependence of the layer thickness of smectic A and smectic B phases of the fd virus show an approximately linear decrease of the layer thickness with increasing concentration. ${ }^{13}$ This indeed is what we obtain for our longest particles. The jumps in layer thickness that we find at the smectic A-to-smectic B phase 

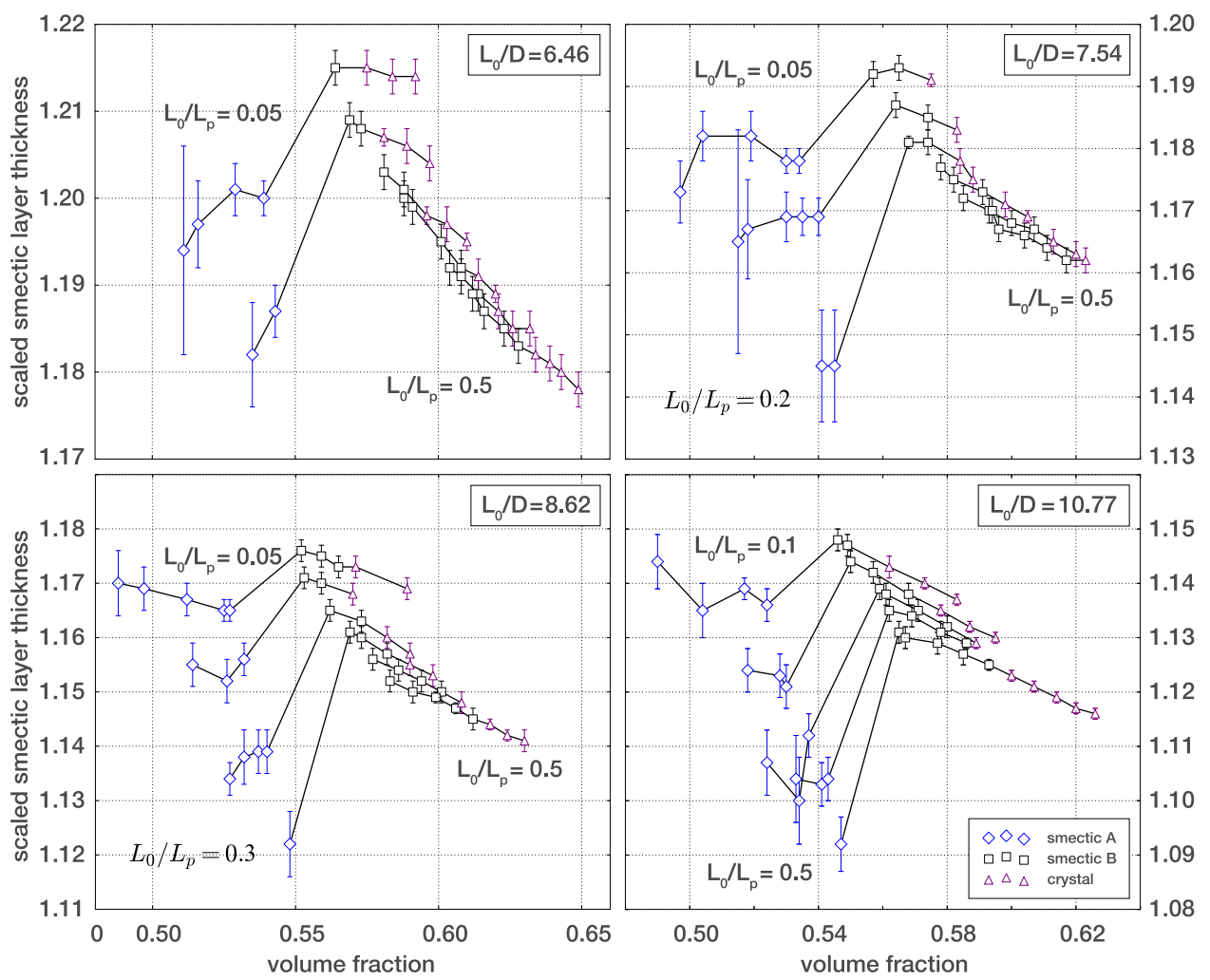

FIG. 8. The scaled smectic layer thickness versus density $\phi$ for various aspect ratios $L_{0} / D$. We calculate the layer thickness from the recipe of Ref. 30. In practice, the layer thickness corresponds to the layer height (rod length) plus to the spacing between layers. The symbols are introduced in Fig. 6. The smectic layer thickness is scaled to the measured average contour length of the particles at that volume fraction. Note the sizeable jump in the layer thickness at the smectic A-to-smectic B phase transition. Furthermore, the layer thickness decreases as a function of the volume fraction for the smectic B and crystal phases. For the smectic A phase, there is a change in the layer thickness behaviour according to the aspect ratio and flexibility. transition are not observed in the experiments on fd viruses. In addition, the actual layer thickness scaled to particle lengths are also smaller for fd viruses than for the particles in our simulations. On the other hand, we should not expect quantitative agreement with measurements on fd virus solutions on account of their much larger aspect ratio.

\section{CONCLUSION}

We perform molecular dynamics simulations to study the influence of flexibility and aspect ratio on the phase behaviour of purely repulsive, rod-like particles. Our particles have aspect ratios between 6 and 11 and ratios of the contour length over the persistence length between 0.05 and 0.5 , i.e., we cover the range from very stiff to slightly flexible particles. By measuring the nematic, smectic, and bond-order parameters and analysing correlation functions, we are able to distinguish five different phases. In order of increasing volume fraction, these include isotropic, nematic, smectic A and B, and crystal phases. Of those phases, we probe the structure of the particles and their arrangement, in particular, in the smectic and crystalline phases.

In agreement with theoretical predictions and previous simulations, we conclude that the isotropic-nematic and nematic-smectic A phase transitions are sensitive functions of the aspect ratio and the flexibility of the particles. For the former, the larger the aspect ratio is, the lower the volume fraction at the transition. For the former and the latter, the larger the flexibility is, the larger the volume fraction at the transition. In fact, the smectic A phase disappears for a sufficiently large ratio of the contour length over the persistence length, which is a measure for the bending flexibility of the particles. We find the transitions to the other more highly ordered phases to be much less influenced by both the aspect ratio and the flexibility of the particles.

On increasing the concentration and going from the isotropic phase through the various liquid-crystalline phases to the crystal phase, we find that the end-to-end distance of the particles increasingly approaches their contour length. This is not entirely surprising because the more strongly ordered the phase is, the more the bending fluctuations are suppressed. In fact, we find, at least for our model bead-chain particles, that bending fluctuations are essentially completely suppressed in smectic B and crystalline phases, explaining the insensitivity of their stability to the persistence length. In other words, the particles in those phases are stretched to their contour length and resemble rigid rods.

The layer spacings that we find in the smectic A, smectic $\mathrm{B}$, and crystal phases exceed the contour length of the particles. Interestingly and counter-intuitively, these layer spacings need not decrease with increasing concentration of particles, at least in the smectic A phase. We find that depending on the aspect ratio and flexibility, spacing may actually increase. This is possible provided that the increasing concentration is more than compensated for by an in-layer density increase. We verified this and not surprisingly, this turns out to be the case in our simulations. We have not been able to pinpoint under what conditions this happens and also do not have an explanation for this phenomenon. Connected to this, we also find that the layer spacing increases upon going from the smectic A to the smectic B phase. This arguably is caused by the increase in free volume across the transition. The layer spacing in the smectic B and crystalline phases does behave as expected, that is, decrease with increasing concentration. 
If we compare our phase diagrams with that of fd viruses in aqueous solution, then all phases are reproduced, except for the columnar phase that for fd viruses occurs for concentrations in between the smectic B and crystal phases is stable. Of course, our particles are much shorter and perhaps it is that suppresses the columnar phase in our simulations. The existence of the columnar phase in dispersions of monodisperse rod-like particles remains somewhat enigmatic and has been the subject of a lot of debate in the literature. ${ }^{35}$ It has been suggested that explicit modeling of the electrostatics stabilises that phase albeit we cannot exclude the possibility that it is a question of a combination of flexibility and large-enough aspect ratio. ${ }^{7}$ The challenge is to reach aspect ratios large enough to investigate this hypothesis.

\section{ACKNOWLEDGMENTS}

This project has received funding from the European Union's Horizon 2020 research and innovation programme under the Marie Skłodwska-Curie Grant Agreement No. 641839 and from HFSP under Grant Agreement No. RGP0017/2012. We thank Eric Grelet for critical reading of the manuscript.

\section{APPENDIX: ONSAGER THEORY OF LINEARLY COMPRESSIBLE HARD RODS}

We observe in our simulations, the contraction of the average contour length of the chains for increasing volume fractions on account of their finite extensional compressibility. At the isotropic-nematic phase transition, there is also a discontinuity in their contour length, with the particles in the nematic phase being slightly longer. A similar discontinuity occurs at the phase transition towards the smectic-B-crystal phase. These two observations can be rationalised with theoretical predictions that we obtain by applying the Onsager theory to extensible rods.

To this end, we consider a system of $N$ bead-spring chains in a volume $V$ at temperature $T$. Each rod consists of $n$ beads connected with $n-1$ harmonic bonds with elastic constant $\kappa$ and rest length $r$. Each chain has total rest length $L_{0}=(n-1) r$ and diameter $D$. The contour length $L$ changes with the concentration of the dimensionless concentration $c=B_{2}^{\text {iso }} \rho$, with $\rho=N / V$ being the number density of particles. The free energy $F$ can be written as a function of the orientational distribution function $f(\Omega)$ and the compression of the chain $x=L / L_{0}$

$$
\frac{F[f]}{N k_{B} T}=A+\ln c+\sigma[f]+c \rho[f] x^{2}+K(x-1)^{2},
$$

where $\mathrm{A}$ is a constant, $\ln c$ is the ideal gas distribution, $\sigma[f]$ is the orientation entropy, $c \rho[f] x^{2}$ is the packing entropy, and $K(x-1)^{2}$ is related with the potential energy of the harmonic springs with

$$
K=\frac{\kappa L_{0}^{2}}{2(n-1) k_{B} T} .
$$

The third and fourth terms mentioned previously are given by the expressions

$$
\sigma[f]=\int f(\Omega) \ln (4 \pi f(\Omega)) d \Omega
$$

and

$$
\rho[f]=\frac{4}{\pi} \int|\sin \gamma| f(\Omega) f\left(\Omega^{\prime}\right) d \Omega d \Omega^{\prime},
$$

where $|\sin \gamma|$ is the angle between the chains with orientation $\Omega$ and $\Omega^{\prime}$.

In the isotropic phase, the normalised distribution function is $f(\Omega)=1 / 4 \pi$, resulting in an orientational entropy $\sigma[f]=0$ and a packing entropy $\rho[f]=1$. The free energy for the isotropic state becomes

$$
\frac{F^{\text {iso }}}{N k_{B} T}=A+\ln c+c x^{2}+K(x-1)^{2} .
$$

The equilibrium condition for $x, \frac{\partial}{\partial x}\left[\frac{F^{\text {iso }}}{N k_{B} T}\right]=0$, leads to the compression in the isotropic phase,

$$
x^{\text {iso }}=\frac{K}{c+K} .
$$

For the nematic phase, we follow a similar procedure as Odijk. ${ }^{16}$ We assume the orientational distribution function to be Gaussian and obeys cylindrical and inversion symmetry as follows:

$$
f(\theta)=\left\{\begin{array}{ll}
\alpha / 4 \pi \exp \left(-\alpha \theta^{2} / 2\right), & \text { if } 0 \leqslant \theta \leqslant \pi / 2 \\
\alpha / 4 \pi \exp \left(-\alpha(\pi-\theta)^{2} / 2\right), & \text { if } \pi / 2<\theta \leqslant \pi
\end{array},\right.
$$

where the normalisation is only accurate for $\alpha \gg 1$. For this distribution, we have for the orientational entropy $\sigma[f] \sim \ln \alpha-1$ and for packing entropy $\rho[f] \sim 4 / \sqrt{\alpha \pi}{ }^{8}$ The free energy for the nematic state is then

$$
\frac{F^{\mathrm{nem}}}{N k_{B} T}=A+\ln c+\ln \alpha-1+\frac{4 c x^{2}}{\sqrt{\alpha \pi}}+K(x-1)^{2} .
$$

From this expression, we find equilibrium values $\alpha=4 c^{2} x^{4} / \pi$ and

$$
x^{\mathrm{nem}}=\frac{1}{2}+\sqrt{\frac{1}{4}-\frac{2}{K}} .
$$

For $x^{\mathrm{nem}}$, there is also a negative root solution that we ignore for being physically unrealistic. For $K \rightarrow \infty, x^{\text {nem }}=1$. For $K<8$, the compression becomes imaginary, meaning that the nematic phase becomes unstable.

Equations (A6) and (A9) describe the behaviour of the mean length of our chains in the isotropic and nematic phases. We now calculate the coexistence concentration. Coexistence between two phases occur when the osmotic pressure $\Pi=-(\partial F / \partial V)_{N, T}$ and chemical potential $\mu=(\partial F / \partial N)_{V, T}$ are equal for both states, $\mu^{\text {iso }}=\mu^{\text {nem }}$ and $\Pi^{\text {iso }}=\Pi^{\text {nem }}$. From these equations, we then calculate the coexistence concentrations for the isotropic $c^{\text {iso }}$ and the nematic phase $c^{\text {nem }}$.

We now add flexibility to our previous model to study how it affects the discontinuity in the average length of the chains at the isotropic-nematic phase transition. Our starting point is the expression derived by Odijk ${ }^{15,16}$ describing the orientational entropy for semi-flexible particles, $L / L_{P} \ll 1$. For the isotropic phase, there is no change of the orientational entropy. For the 


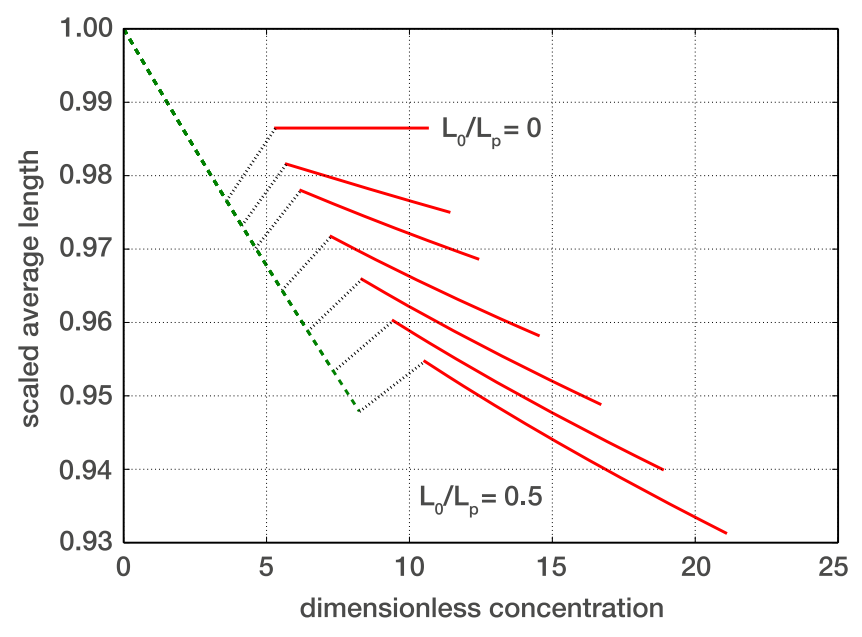

FIG. 9. Scaled average length of the chains $x=L / L_{0}$ as a function of the dimensionless concentration $c$, for $K=150$ and flexibilities $L_{0} / L_{P}=0,0.05$, $0.1,0.2,0.3,0.4$, and 0.5 . The green dashed line represents the isotropic phase, the red lines represent the nematic phase, and the black dots connect the points of coexistence.

nematic phase, there is the extra term $\sigma^{\mathrm{Odijk}}=L_{0} \alpha x / 4 L_{P}$, and then the orientational entropy is

$$
\sigma=\ln \alpha-1+\frac{L_{0} \alpha x}{4 L_{P}}
$$

With this new orientational entropy, the free energy for the nematic phase becomes

$$
\frac{F^{\text {nem }}}{N k_{B} T}=A+\ln c+\ln \alpha-1+\frac{L_{0} \alpha x}{4 L_{P}}+\frac{4 c x^{2}}{\sqrt{\alpha \pi}}+K(x-1)^{2} .
$$

Solving the equilibrium value for $\alpha$ and $x$, we obtain the compression of the chain as a function of the dimensionless concentration. These and the coexistence concentrations are calculated numerically.

Finally, we compare the simulations with the model calculations. We specifically perform the calculations for $K=150$ and for flexibilities $L_{0} / L_{P}=0,0.05,0.1,0.2,0.3,0.4$, and 0.5 , as can be seen in Fig. 9. These values coincide with the simulated values, except $L_{0} / L_{P}=0$, the rigid rod limit that we did not simulate. Despite the approximations of our model, we find qualitative similarities between these results and our simulation results. First, the decrease of the average length of the chain with increasing concentration for both isotropic and nematic phases. Second, the discontinuity in the average length decrease with the increase of flexibility.

${ }^{1}$ L. Onsager, Ann. N. Y. Acad. Sci. 51, 627 (1949).

${ }^{2}$ A. R. Khokhlov and A. N. Semenov, Phys. A 108, 546 (1981).

${ }^{3}$ A. R. Khokhlov and A. N. Semenov, Phys. A 112, 605 (1982).

${ }^{4}$ J. V. Selinger and R. F. Bruinsma, Phys. Rev. A 43, 2910 (1991).

5 J. V. Selinger and R. F. Bruinsma, Phys. Rev. A 43, 2922 (1991).

${ }^{6}$ A. V. Tkachenko, Phys. Rev. Lett. 77, 4218 (1996).

${ }^{7}$ P. van der Schoot, J. Phys. II 6, 1557 (1996).

${ }^{8}$ G. J. Vroege and H. N. W. Lekkerkerker, Rep. Prog. Phys. 55, 1241 (1992).

${ }^{9}$ A. Kuijk, D. V. Byelov, A. V. Petukhov, A. van Blaaderen, and A. Imhof, Faraday Discuss. 159, 181 (2012).

${ }^{10}$ Z. Dogic and S. Fraden, Phys. Rev. Lett. 78, 2417 (1996).

${ }^{11}$ E. Grelet, M. P. Lettinga, M. Bier, R. van Roij, and P. van der Schoot, J. Phys.: Condens. Matter 20, 494213 (2008).

${ }^{12}$ E. Grelet, Phys. Rev. Lett. 100, 168301 (2008).

${ }^{13}$ E. Grelet, Phys. Rev. X 4, 021053 (2014).

${ }^{14}$ E. Grelet and R. Rana, Soft Matter 12, 4621 (2016).

${ }^{15}$ T. Odijk, Polym. Commun. 26, 197 (1985).

${ }^{16}$ T. Odijk, Macromolecules 19, 2313 (1986).

${ }^{17}$ Z. Y. Chen, Macromolecules 26, 3419 (1993).

${ }^{18}$ R. C. Hidalgo, D. E. Sullivan, and J. Z. Y. Chen, Phys. Rev. E 71, 041804 (2005).

${ }^{19}$ K. Shundyak, R. van Roij, and P. van der Schoot, Phys. Rev. E 74, 021710 (2006).

${ }^{20}$ M. Dijkstra and D. Frenkel, Phys. Rev. E 51, 5891 (1995).

${ }^{21}$ S. C. McGrother, D. C. Williamson, and G. Jackson, J. Phys. Chem. 104, 6755 (1996).

${ }^{22}$ P. Bladon and D. Frenkel, J. Phys.: Condens. Matter 8, 9445 (1996).

${ }^{23}$ G. Cinacchi and L. de Gaetani, Phys. Rev. E 77, 051705 (2008).

${ }^{24}$ S. Naderi and P. van der Schoot, Phys. Rev. E 88, 032307 (2013).

${ }^{25}$ S. Naderi and P. van der Schoot, J. Chem. Phys. 141, 124901 (2014).

${ }^{26}$ S. A. Egorov, A. Milchev, P. Virnau, and K. Binder, Soft Matter 12, 4944 (2016).

${ }^{27}$ R. van Roij, P. Bolhuis, B. Mulder, and D. Frenkel, Phys. Rev. E 52, R1277 (1995).

${ }^{28}$ S. Plimpton, J. Comput. Phys. 117, 1 (1995).

${ }^{29}$ P. G. de Gennes and J. Prost, The Physics of Liquid Crystals (Oxford University Press, 1995).

${ }^{30}$ J. M. Polson and D. Frenkel, Phys. Rev. E 56, 6260 (1997).

${ }^{31}$ D. R. Nelson and B. I. Halperin, Phys. Rev. B 19, 2457 (1979).

${ }^{32}$ W.-K. Qi, S.-M. Qin, X.-Y. Zhao, and Y. Chen, J. Phys.: Condens. Matter 20, 245102 (2008).

${ }^{33}$ P. Bolhuis and D. Frenkel, J. Chem. Phys. 106, 666 (1997).

${ }^{34}$ M. R. Wilson, Mol. Phys. 81, 675 (1994).

${ }^{35}$ H. H. Wensink, J. Chem. Phys. 126, 194901 (2007). 